\title{
Clínica Analítico-Comportamental no Brasil: Histórico, Treinamento e Supervisão
}

\section{Behavior-Analytical Therapy in Brazil: Background, Training and Supervision

\author{
Actuación en Psicología Clínica Analítico-Conductual en Brasil: Historia, \\ Formación y Supervisión
}

Renatha Rafihi-Ferreira ${ }^{3}$, Deivid Regis dos Santos², Felipe Alckmin-Carvalho ${ }^{3}$, Maria Rita Zoega Soares ${ }^{4}$

$[1,3]$ Universidade de São Paulo [2,4] Universidade Estadual de Londrina I Título abreviado: Clínica Analítico-Comportamental I Endereço para correspondência: Universidade de São Paulo, Instituto de Psicologia. Av. Prof. Mello Moraes, 1721, Prédio F, Sala 19. Butantã. CEP 05508030 - São Paulo, SP - Brasil. I Email: rerafihi@usp.br I DOI: 10.18761/pac.2016.001

Resumo: O Brasil é um dos países que mais se destaca na formação clínica de analistas do comportamento. No presente estudo é apresentado um breve histórico da terapia analítico-comportamental, com seu surgimento e evolução da área no Brasil, incluindo as terapias de modificação do comportamento, com base filosófica no Behaviorismo Metodológico, até as Terapias de Terceira Onda, fundamentadas no Behaviorismo Radical. Ressalta-se a importância de habilidades do terapeuta ao atendimento clínico, com a discussão de questões relacionadas ao desenvolvimento de vínculo genuíno e positivo com o cliente, observação, identificação, descrição de como operacionalizar e analisar funcionalmente os comportamentos problema. São apresentadas considerações sobre a relação supervisor-supervisionando e sobre o papel do supervisor na aquisição de repertórios básicos na formação do clínico iniciante. Por fim, são descritos recursos que podem ser utilizados pelo supervisor para facilitar o desenvolvimento de habilidades em seus supervisionandos, como o ponto de escuta eletrônico, a observação direta a partir de salas com espelho unidirecional e as gravações de áudio e vídeo. Espera-se que esse artigo seja útil para práticos da clínica analítico-comportamental e sirva também como recurso didático para professores das disciplinas de Análise do Comportamento na graduação de universidades brasileiras.

Palavras-chave: psicologia clínica, análise do comportamento, terapia comportamental, treinamento e supervisão 
Abstract: Brazil stands out as one of the best countries offering clinic education for behavior analysts. This study presents a brief history of analytical-behavioral therapy, from its birth and evolution in Brazil to behavior conditioning therapies, philosophically based on Methodological Behaviorism, including even Third Wave Therapies, grounded on Radical Behaviorism. It's important to mention the therapist's skills in clinical work, discussing issues related to the genuine and positive bond developed with the client when observing, identifying, describing how to functionally operate and analyze problem behavior. Considerations about the relationship between supervisor and supervised students are presented, as well as about the supervisor's role when acquiring basic knowledge in the study of clinical therapy. Lastly, some resources are described that can be used by the supervisor to help with the skills development of their supervised students, such as the electronic listener, the direct observation from unidirectional mirror rooms and audio and video recordings. It is expected that this article will be useful for analytical behavioral clinic practitioners and can also be used as a didactic resource for professors of Behavioral Analysis subjects for undergraduate degree students in Brazilian Universities.

Keywords: clinical psychology, behavior analysis, behavior therapy, training and supervision.

Resumen: Brasil destaca por su formación clínica en el Análisis Conductual. Se expone un breve desarrollo histórico de la terapia basada en el análisis conductual, desde su nacimiento, abarcando las terapias de modificación de la conducta con base filosófica, el conductismo metodológico y las terapias de tercera generación, fundamentadas en el Conductismo radical. Se destaca la importancia de la alianza terapéutica cimentada en el desarrollo de un vínculo auténtico y positivo entre el terapeuta y el paciente, así como las habilidades de observación, identificación, descripción, operativización de las variables y el análisis funcional de la conducta problema. Se ofrecen fundamentos sobre la relación supervisor-supervisado y sobre la función del supervisor en la adquisición del repertorio conductual básico en la formación del supervisado. Finalmente se describen los recursos utilizados por el supervisor para facilitar el desarrollo de habilidades en sus supervisados, como el intercomunicador intrauricular, la observación directa a través del espejo unidireccional y las grabaciones de audio y vídeo. Se espera que este articulo sea útil para los iniciados y como recurso educativo para los profesores universitarios, en el análisis funcional de la conducta clínica.

Palabras-clave: Psicología clínica, análisis conductual, terapia conductual, capacitación y supervisión. 
Ainda que não haja consenso sobre a época precisa de surgimento da Psicoterapia Comportamental, a maioria dos autores concorda que muitas modificações ocorreram desde que esta se tornou reconhecida como uma forma de intervenção terapêutica (Silvares, 1991). O provável primeiro registro foi feito em um trabalho para modificação do comportamento de pacientes psiquiátricos adultos (Linsdley, Skinner, \& Salomon, 1953), com sustentação filosófica no Behaviorismo Metodológico e intervenções com foco na eliminação de comportamentos indesejados (que produziam risco ou prejuízos aos próprios indivíduos ou à comunidade). Nessa fase inicial os esforços do terapeuta estavam voltados para modificação e não para a avaliação funcional dos comportamentos considerados problema (D’Zurilla \& Goldfried, 1971).

Para abordarmos a Psicoterapia Comportamental, faz-se necessário retomar o desenvolvimento do Behaviorismo Radical, a filosofia da ciência do comportamento, porque tanto a filosofia do comportamento, quanto a ciência experimental e a análise aplicada são áreas inter-relacionadas que se mantêm em um processo contínuo de alimentação recíproca (Tourinho, 1999). O desenvolvimento da área foi motivado pela busca de uma psicologia científica, que considerasse o comportamento como objeto de estudo a partir de uma concepção naturalista, monista, objetivista e evolucionista dos eventos psicológicos. Isso só foi possível a partir do desenvolvimento das pesquisas em fisiologia na Rússia, do surgimento do Behaviorismo nos Estados Unidos e do desenvolvimento da Psicologia da Aprendizagem (Kazdin, 1985).

Os trabalhos de Pavlov (1849-1936), no início do século XX, estabeleceram os princípios do condicionamento respondente (Millenson, 1975; Pavlov, 1928), enquanto que os de Thorndike (1874-1949), estabeleceram a Lei do Efeito, base do condicionamento operante (Thorndike, 1898; Millenson, 1975). Watson (1878-1958), cujos estudos fundamentaram o paradigma de condicionamento respondente, apresentou contribuições relevantes à Psicologia Comportamental por meio da publicação do artigo "Psychology as the Behaviorist views it”, em 1913 (Carrara, 2005; Watson, 1913).

Skinner (1904-1990) fez suas primeiras publicações na década de 1930 (Skinner, 1935; Skinner,
1937), apresentando o paradigma do comportamento operante, que constituiu uma contribuição essencial para a evolução da Psicoterapia Comportamental, visto que até o momento predominavam estudos envolvendo o paradigma respondente, com alcance limitado para o tratamento de comportamentos complexos (Carrara, 2005; Richelle, 1993).

Com o avanço do estudo do comportamento operante, por meio de Skinner, foi possível tornar-se aplicada a teoria da Análise do Comportamento em um âmbito clínico. A Análise do Comportamento aplicada à clínica segue uma perspectiva monista e os comportamentos considerados problemáticos e com necessidade de modificação, são analisados e compreendidos a partir dos mesmos princípios presentes em qualquer outro comportamento, uma vez que são aprendidos e mantidos da mesma forma (Sant'Anna \& Gongora, 1987).

$\mathrm{Na}$ conduta do clínico analítico-comportamental, avaliação e intervenção são processos entrelaçados que ocorrem durante todo o processo terapêutico. É necessário primeiro identificar o comportamento-problema e buscar os antecedentes e consequentes que selecionaram e que mantêm o comportamento, selecionar a intervenção e avaliar continuamente seus efeitos (Silvares \& Range, 2001). É importante que o cliente aprenda a realizar análise funcional do seu próprio comportamento, identificando as contingências ambientais determinantes de suas queixas, para alcançar resultados psicoterapêuticos duradouros (Barcellos \& Haydu, 1998).

A partir da década de 1980, autores como Kohlenberg e Tsai (2001) argumentaram que a relação terapêutica também deve ser analisada em termos funcionais. Na mesma direção, Hayes, Strosahl, \& Wilson (1999) defenderam a análise de comportamentos privados em termos funcionais, bem como a análise contextual de processos cognitivos e da linguagem. Essas novas modalidades de Terapias Comportamentais Clínicas, denominadas de Terapias de Terceira Onda, incorporam as aquisições técnicas da Terapia Comportamental Clássica e da Terapia Cognitivo-Comportamental, mas entendem a mudança terapêutica, anteriormente atribuída a processos de condicionamento clássico ou à reestruturação de esquemas cognitivos (Terapia Cognitivo-Comportamental), em termos 
behavioristas radicais, enfocando as contingências operantes interpessoais e o efeito exercido por contextos socioverbais (Souza \& Vanderberghe, 2007).

A Terceira Onda engloba quatro diferentes modalidades de psicoterapias: (a) Psicoterapia Analítico-funcional - FAP - (Kohlenberg \& Tsai, 2001), (b) Terapia de Aceitação e Compromisso - ACT - (Hayes, 1987; Hayes, Strosahl, \& Wilson, 1999), (c) Terapia Comportamental Integrativa de Casal (Jacobson, 1992) e (d) Terapia Comportamental Dialética (Linehan, 1993). Essas psicoterapias estão comprometidas com a experiência direta acima da compreensão racional, e com a promoção de aceitação de eventos privados aversivos, ao invés do controle, e enfatizam as trocas espontâneas entre terapeuta e cliente como elementos-chave do processo terapêutico (Vandenberghe, 2002; Vandenberghe, 2007).

Conte (2010) aponta que, de um ponto de vista histórico, as Terapias de Terceira Onda constituem o retorno da filosofia behaviorista na prática clínica através de uma releitura do processo terapêutico em termos contextualistas. A autora aponta ainda que esses processos se propõem a afetar classes comportamentais mais amplas, via análises funcionais molares (por exemplo, de comportamentos relacionados à forma como os pacientes lidam com seu sofrimento), ao invés de trabalhar com queixas menores, por meio de análises funcionais moleculares.

As psicoterapias de Terceira Onda têm alcançado resultados promissores no tratamento de diversos problemas de comportamento e transtornos psiquiátricos, tanto internacionalmente (eg.: Callaghan et al., 2012; Cattivelli, Tirelli, Berardo, \& Perin, 2012) como no Brasil (eg.: Oshiro, Kanter, \& Meyer, 2012; Pezzato, Brandão, \& Oshiro, 2012).

Considera-se que o desenvolvimento histórico da Psicologia Comportamental no Brasil foi bastante peculiar. Constata-se que desde o início, houve adesão por parte dos clínicos à análise Skinneriana e a utilização de técnicas do paradigma respondente, sem necessidade de utilização de explicações internalistas. A seguir será apresentado um breve histórico da Psicoterapia Comportamental no Brasil, que suscitou tal discussão.

\section{Breve Histórico da Psicoterapia Comportamental no Brasil}

O livro "Princípios de Psicologia" (Keller \& Schoenfeld, 1950/1968) foi um marco na história da Análise do Comportamento, tendo preparado o terreno para "Ciência e Comportamento Humano" (Skinner, 1953/1967). Essas obras foram as primeiras na área a serem traduzidas para o português e cruciais para a introdução do Behaviorismo no Brasil (Todorov \& Hanna, 2010).

Fred S. KeIler veio ao Brasil em 1961 e iniciou cursos e atividades no Departamento de Fisiologia na Universidade de São Paulo (USP), com a participação dos professores Rodolfo Azzi e Carolina Bori e das alunas Maria Amélia Matos e Maria Inês Rocha. Naquele ano, só a Pontifícia Universidade Católica do Rio de Janeiro (PUC-RJ) e a USP ofereciam o curso de graduação em Psicologia, iniciados os dois em 1958 (Todorov \& Hanna, 2010).

Em 1961, com a contribuição dos professores Isaías Pesotti e Geraldina Porto Witter, que lecionavam na Faculdade de Filosofia Ciências e Letras de Rio Claro, houve a inserção da Análise Experimental do Comportamento (AEC). Nesse ano foi criado o primeiro laboratório de AEC no Brasil, no curso de Pedagogia. No ano seguinte, o professor americano behaviorista John Gilmour Sherman, também veio ao Brasil, o que ajudou a incentivar as atividades neste laboratório. Tal condição auxiliou na constituição do curso de Psicologia da Universidade de Brasília (UnB), com a contribuição direta de analistas do comportamento como João Cláudio Todorov, Alvaro Duran e Luiz Otavio de Seixas Queiroz (Cesar, 2002).

Os cursos para formação do psicólogo foram regulamentados pela Lei 4.119 de 27 de agosto de 1962, dia em que se comemora o dia do psicólogo. O primeiro centro de formação de analistas do comportamento foi a Universidade de Brasília, sob o comando de Carolina Bori. Na Faculdade de Filosofia, Ciências e Letras de Ribeirão Preto, hoje Universidade de São Paulo (USP), o curso de Psicologia começou a funcionar em março de 1964 (Todorov \& Hanna, 2010). No ano de 1965 houve dissipação na estrutura da UnB em função do golpe militar ocorrido no país (Rubiano, n.d.). 
Em 1969, a professora Rachel Rodrigues Kerbauy, aluna do professor Sherman, levou a AEC (Análise Experimental do Comportamento) e ACC (Análise Comportamental Clínica) para a Faculdade Sedes Sapientiae ${ }^{1}$, dando início ao curso. Em fins de 1969, o professor João Claudio Todorov voltou dos Estados Unidos, onde fizera seu doutoramento e iniciou as atividades de grupo de pesquisa na Faculdade de Medicina de Ribeirão Preto, hoje a atual USP-RP. Tais atividades e a proveitosa interação com o grupo da Psicofarmacologia liderado pelo Dr. Frederico G. Graeff favoreceram a ocorrência de discussões científicas relevantes (César, 2002).

Após este período, na década de 1970, os professores João Cláudio Todorov e Thereza Mettel foram para a UnB e a professora Carolina Bori retornou a São Paulo, desenvolvendo na USP (Campus de São Paulo e de Ribeirão Preto) e na Universidade Federal de São Carlos (UFSCAR) um dos maiores núcleos de formação em Análise do Comportamento (Todorov \& Hanna, 2010).

Assim, no final da década de 60 e início dos anos 70, começaram a atuar no mercado de trabalho, os primeiros psicólogos formados em conformidade com a nova legislação, com seus direitos e obrigações devidamente regulamentados, ao lado de outros profissionais que já atuavam na área. Coube a estes novos profissionais redefinirem, por meio de sua atuação, o campo de trabalho da Psicologia em diferentes situações, relacionadas à atuação profissional, ensino e pesquisa. Algumas entidades congregavam profissionais da área como a Associação Brasileira de Psicólogos, a Associação Brasileira de Psicologia Aplicada, a Sociedade de Psicologia de São Paulo e a Sociedade Mineira de Psicologia. Neste período, ainda não existiam os órgãos de classe, muito menos os sindicatos da área, que pudessem orientar, apoiar os psicólogos e lutar pela profissão. As Reuniões Anuais da Sociedade Brasileira para o Progresso da Ciência (SBPC) eram o principal fórum para conhecer e debater os trabalhos científicos de Psicologia (Rubiano, n.d.).

A Sociedade de Psicologia de Ribeirão Preto (SPRP) em 1971, hoje chamada Sociedade Brasileira

1 A partir de 1975 passou a se chamar Instituto Sedes Sapientiae. de Psicologia (SBP), auxiliou na consolidação da profissão no país por meio da atuação de nomes como, Reinier Rozestraten, João Cláudio Todorov, Angela Simões Rozestraten, Ricardo Gorayeb, Terezinha Moreira Leite, José André Jacquemin, José Lino de Macedo, Luiz Marcellino de Oliveira, Zélia Biasoli Alves, Maria Clotilde Rossetti Ferreira, Isaias Pessoti, Deysy das Graças de Souza, José Aparecido da Silva, entre outros (Gorayeb, 1990; Rozestraten, 1988; Souza \& Alves, 1988).

Como ainda não existia o Conselho Federal de Psicologia nem os Conselhos Regionais, a Sociedade de Psicologia foi considerada mais do que um órgão de comunicação científica, mas também um órgão fiscalizador da ética profissional. Em fevereiro de 1972, a lei 5.766 de 20/12/1971 criou o Conselho Federal e os Conselhos Regionais de Psicologia. Nesse período ocorrem discussões sobre a atividade profissional do psicólogo clínico. Em 1973, quatorze entidades elaboraram um programa de ação em defesa dos direitos dos psicólogos assegurados pela lei 4119/62 e convocação de reunião para dar forma mais concreta à Lei 5.766 de 20/12/71, que criou o Conselho Federal e os Conselhos Regionais de Psicologia (Gorayeb, 1990; Rozestraten, 1988; Souza \& Alves, 1988).

Neste período, a professora Thereza Mettel ministrou na Universidade de São Paulo o primeiro curso de Modificação de Comportamento, em nível de pós-graduação no Brasil. Paralelamente, um grupo de professores (Hélio Guilhardi, Maria do Carmo Guedes, Luís Otávio de Seixas Queiroz, José Ernesto Bolonha) da Pontifícia Universidade Católica de São Paulo (PUC-SP) desenvolveu um curso de treinamento em Modificação do Comportamento, com a participação do professor Gary Martin. Foi neste contexto, em 1974 no estado de São Paulo, sob influência do professor Gary Martin, que este grupo criou a primeira associação comportamental no Brasil, que recebeu o nome de Associação de Modificação de Comportamento (AMC), no qual visava atender ao profissional em Análise do Comportamento (Botomé, 2006; César, 2002).

Posteriormente, no ano de 1985, buscando ampliar e continuar os trabalhos da AMC e com o objetivo de abarcar as contribuições da Análise do Comportamento tanto em nível de pesquisa básica como aplicada, a proposta da associação 
foi reafirmada passando de uma sociedade local (São Paulo) para nacional, surgindo a Associação Brasileira de Análise do Comportamento (ABAC) (Botomé, 2006).

Até os anos 90, se consolidaram os cursos da Pontifícia Universidade Católica de São Paulo (PUC-SP), da Universidade Federal de São Carlos (UFSCar), da Universidade Federal do Pará (UFPA), da Universidade Federal de Santa Catarina (UFSC) e da Universidade Estadual de Londrina (UEL), todas influenciadas direta ou indiretamente por São Paulo e/ou Brasília. A formação básica do psicólogo brasileiro sofreu grande influência da atuação de Carolina Bori, principal responsável pela criação e disseminação de laboratórios de ensino em psicologia experimental no país. Graças aos seus esforços, todos os cursos de graduação de grandes centros de formação passaram a incluir em seus currículos, e isto é mantido até hoje, o conteúdo de psicologia como ciência natural, a preocupação com a observação sistemática e os métodos de investigação científica, bem como demonstrações, em atividades de laboratório, de manipulação e controle de variáveis ambientais e de seus efeitos sobre o comportamento (Todorov \& Hanna, 2010).

No ano de 1990, os contatos iniciais que decorreram da aproximação de psicoterapeutas cariocas, suscitaram aproximações com os grupos de São Paulo (liderado por Rachel Kerbauy), Campinas (Hélio Guilhardi), Ribeirão Preto (Ricardo Gorayeb e Vera Otero) com o objetivo de constituir uma Associação Brasileira de Psicoterapia Comportamental. No ano seguinte houve a fundação por delegação, pelo grupo do Rio de Janeiro, da Associação Brasileira de Psicoterapia e Medicina Comportamental (ABPMC), com Bernard Rangé e Geraldo Lanna como presidente e vice presidente respectivamente. Desde a década de 1990 até os tempos atuais, a ABPMC promove encontros anuais e tem recebido grandes contribuições de pesquisadores como Sonia Beatriz Meyer, Roberto Alves Banaco, Edwiges Ferreira de Mattos Silvares, Maly Delitti, Vera Raposo do Amaral, Hélio José Guilhardi, Luc Vandenberghe, Regina Christina Wielenska, entre outros. Estes profissionais têm desenvolvido pesquisas na área clínica, sendo pessoas de referência na formação de terapeutas comportamentais.
Atualmente, analistas do comportamento publicam a maior parte de sua produção em periódicos nacionais. Pesquisas básicas e aplicadas e trabalhos de intervenção têm sido divulgados em revistas gerais de psicologia, especialmente a Psicologia: Teoria e Pesquisa, Psicologia: Reflexão e Crítica, Temas em Psicologia e Psicologia (USP) e em revistas especializadas em Análise do Comportamento. O número de periódicos que publicam especificamente artigos teóricos e empíricos de análise do comportamento em língua portuguesa cresceu na última década. A primeira a ser criada foi a Revista Brasileira de Terapia Comportamental e Cognitiva, publicada pela ABPMC, criada por Rachel Kerbauy em 1999. A Revista Brasileira de Análise do Comportamento publica também artigos na língua inglesa e foi criada por João Claudio Todorov em 2005. Recentemente, o Núcleo Paradigma iniciou sua publicação intitulada Perspectivas em Análise do Comportamento. A Acta Comportamentalia é um periódico da Universidad de Guadalajara que publica artigos em línguas latinas, do qual Maria Amélia Matos foi a primeira editora de língua portuguesa. A série Sobre Comportamento e Cognição, inicialmente organizada por Roberto Alves Banaco, se tornou um periódico (Todorov \& Hanna, 2010). Tais publicações têm influenciado discussões e fazem parte do conteúdo programático de disciplinas da própria formação do analista comportamental.

\section{Formação do Analista do Comportamento}

Constata-se hoje que, na graduação, a Análise do Comportamento é abordada em disciplinas como Psicologia Geral e Experimental e Psicologia da Aprendizagem. Em algumas instituições é ministrada em disciplinas práticas como Psicologia Clínica, Psicologia do Excepcional e de forma mais incipiente nas áreas de Psicologia da Saúde, Psicologia Organizacional, Psicologia Social e Psicologia do Esporte.

Frequentemente a grade curricular está baseada, predominantemente, nos pressupostos básicos do Behaviorismo Metodológico, o que suscita críticas infundadas à Análise do Comportamento e resistência de discentes, que veem pouca aplica- 
bilidade desses conteúdos à clínica de crianças e adultos com desenvolvimento típico. As Terapias de Terceira Onda, por exemplo, são pouco exploradas, e em muitas universidades, nem sequer apresentadas. Outro ponto que merece destaque é que, infelizmente, conteúdos de Análise do Comportamento são ministrados em alguns cursos de Psicologia ou outras áreas, por professores não familiarizados com os pressupostos desta ciência, o que pode gerar no aluno, percepção equivocada sobre a proposta (Todorov \& Hanna, 2010).

Embora a formação em Análise do Comportamento muitas vezes não seja aprofundada na graduação, é adequadamente estruturada na pós-graduação stricto e lato sensu. Alguns cursos trabalham com programas exclusivos na linha teórica da Análise do Comportamento (Programa de Análise do Comportamento - Universidade Estadual de Londrina; Programa de Psicologia Experimental: Análise do Comportamento da PUC-SP; Programa de Pós-Graduação em Ciências do Comportamento da UnB) e outros, em áreas mais abrangentes, oferecendo possibilidade de orientação de trabalhos em Análise do Comportamento (Programa de Pós-Graduação em Psicologia da Universidade Federal do Paraná; Programa de Pós-graduação em Psicologia do Desenvolvimento e Aprendizagem da UNESP no Campus de Bauru, dentre outros).

Está claro que algumas habilidades são necessárias para o atendimento do terapeuta comportamental. Atividades relacionadas à supervisão de estágio e a disciplinas aplicadas podem favorecer o desenvolvimento do repertório requerido do clínico para produzir mudanças profundas e duradouras no atendimento de seus clientes. Entende-se por habilidades, comportamentos que podem ser aprendidos por profissionais para desempenhar sua função. Algumas destas habilidades são apontadas pela literatura como observar, analisar funcionalmente, reforçar diferencialmente, manter contato visual, adequar linguagem à do cliente, solicitar e dar informações, agir eticamente, dar orientações e ser empático (Abreu-Motta, De-Farias, \& Coelho, 2010; Falcone, 1999; Meyer \& Vermes, 2001; Ulian, 2007).

Entre os comportamentos requeridos, a observação é um dos requisitos que permite o desenvolvimento da principal habilidade que caracteriza um terapeuta comportamental: realizar análise funcional (Guilhardi, 1988; Silvares, 1997). O ensino da análise funcional é a peça fundamental do clinico analítico comportamental, pois é a partir de uma análise cautelosa da função do comportamento é que torna-se possível escolher as técnicas de intervenção mais eficazes para mudança de determinado comportamento. Por meio da relação com o cliente ou pelo relato verbal deste, torna-se possível identificar e descrever contingências que estão em operação. Ademais, para condução desta análise, é importante identificar e descrever informações relevantes do ambiente do cliente (Guilhardi, 2001).

Para ter acesso a essas informações o aluno precisa entrar em contato direto com o cliente. Sendo assim, precisa aprender como realizar este contato e extrair informações relevantes por meio da observação e do relato verbal. Pensando em como ensinar esta habilidade durante a formação, Silvares e Gongora (2006) propõem um treinamento em entrevista clínica como alternativa para amenizar o problema da introdução brusca na prática da psicoterapia. O treino sugerido pelas autoras deve conter simulação de entrevistas com diferentes tipos de queixas em que um aluno possa fazer o papel do cliente e outro do terapeuta. Nestas condições o feedback deve ser fornecido ao aluno imediatamente após a simulação. O objetivo desta proposta é desenvolver habilidades fundamentais para um clínico, como postura empática, comportamentos não verbais adequados, controle da entrevista, formulação de questões abertas e fechadas, operacionalização das informações, pedidos de esclarecimentos e complementação, parafrasear, análise de sentimentos e sumariar.

Ao realizar entrevistas e relatar sua experiência, o aluno tem a oportunidade de receber feedback sobre os comportamentos apresentados. Tais atividades permitem que o aluno tenha uma experiência de psicoterapeuta antes de entrar em contato com o atendimento clinico propriamente dito. Além disso, possibilita que o aluno descubra previamente alguns déficits comportamentais e busque saná-los (Gongora, 1999). Para nortear este processo, há um modelo de checklist, desenvolvido por Gongora (1999) a fim de guiar o feedback fornecido ao aluno que contém 36 itens que descrevem os comportamentos do entrevistador. 
Entre as variáveis mais importantes para a produção de exposição e ampliação do repertório comportamental do cliente está a capacidade do terapeuta propiciar condições para formação de vínculo legítimo com o cliente (Follette, Naugle, \& Callaghan, 1996). Esse dado é verificado em pesquisas de resultado que associam o desfecho do tratamento à qualidade da relação terapêutica. Além disso, pesquisas de processo ainda estão em andamento para tentar elucidar a associação entre essas variáveis (Prado \& Meyer, 2004).

$\mathrm{O}$ vínculo entre terapeuta e cliente é facilitado pela empatia por parte do clínico. A empatia é uma habilidade importante não só para o terapeuta comportamental, mas para os psicólogos de modo geral. Para ser empático, o profissional deve perceber facilmente as emoções daquele com quem interage (Palhoço, 2011). Por isso, torna-se necessário o desenvolvimento de atividades curriculares que favoreçam a percepção de comportamentos não verbais como gestos e expressões faciais.

Falcone (1999) avaliou o efeito de um programa de treinamento de empatia em dez graduandos do curso de Psicologia. A intervenção foi composta por 11 encontros com duas horas de duração, abordando os seguintes conteúdos: instrução sobre como se comportar empaticamente em situações de ajuda e conflito; identificação das variáveis que dificultam o ouvir e compreender os outros; imaginação de situações em que os participantes interagiam com outras pessoas e; encenações de situações em que os participantes tentavam ser empáticos. Os resultados apontaram eficácia no desenvolvimento do comportamento empático e na generalização desta habilidade para outros contextos.

O desenvolvimento dessas habilidades pode ser facilitado quando o aluno está em supervisão. Esta é parte essencial do ensino-aprendizagem para a formação dos psicólogos. É durante as supervisões que o aluno vivencia a prática relativa à teoria e recebe orientações e feedbacks acerca de seu desempenho, a fim de ampliar e aprimorar seu conhecimento e treinar habilidades e competências para a prática profissional (Witter, 2006)

\section{O papel da supervisão na formação}

A supervisão clínica constitui-se crucial na formação de psicoterapeutas, tendo como objetivo orientar a aplicação de estratégias embasadas teoricamente, estabelecer critérios de conduta ética e promover o desenvolvimento de habilidades clínica (Beckert, 2002; Moreira, 2003).

A formação do terapeuta deve ser compreendida como um processo e não somente como a transmissão de conteúdos, pois para atuar como clínico, de acordo com Vandenberghe (2014) o profissional deve saber o que fazer, como fazer e estar presente em sua atuação, não somente aplicando técnicas e teorias. A partir destes três fatores o supervisor exerce diferentes papéis para contribuir no desenvolvimento do aprendizado do supervisionado.

Neste contexto aprender o que fazer refere-se a um domínio técnico em que o supervisor pode indicar textos, manuais e utilizar da dramatização para o aluno treinar a intervenção antes de aplicá-la. Após esta etapa o supervisor oferece um feedback para o supervisionando em relação ao que aprendeu. Aprender como fazer vai além do conhecimento do que fazer, pois é neste momento que o aprendiz lida com as consequências da sua atuação. O papel do supervisor nesta fase vai além das instruções, tendo função de explorar junto ao aluno como e porque atua desta forma. Estar presente na atuação envolve os sentimentos e valores do clínico, sendo assim não podem ser ensinados como no primeiro nível, nem debatidos, como no segundo. Neste nível é que entra a relação do supervisor com o aprendiz, em uma esfera vivencial, isto é, neste nível o aprendizado é atual, ao vivo e pode acrescentar uma nova perspectiva na supervisão (Vandenberghe, 2014).

Para atingir esses três fatores de aprendizado é importante definir os comportamentos que um terapeuta comportamental deve apresentar e buscar desenvolvê-los a partir de um planejamento de ensino que envolve várias etapas. Após definir os comportamentos alvo, o supervisor deve decompor estes comportamentos em unidades menores, avaliar quais destes comportamentos o aluno já apresenta, definir quais meios/instrumentos irá utilizar para desenvolvê-los e somente posteriormente aplicar sua intervenção (Mattana, 2004). 
As discussões de textos, o treino em análise de contingências e o atendimento clínico com supervisão devem compor o rol de atividades que devem estar presentes na formação dos terapeutas de abordagem comportamental (Ulian, 2007). Para estruturar este processo, Mayer, Nascimento, Sartor, Sabbag, Barbosa, Brandenburg, et al (2014) propõem a realização de uma supervisão antes da sessão, com discussão em grupo sobre o seu preparo. No decorrer da sessão os alunos são observados pelo espelho e suas habilidades e análises funcionais são registradas em uma ficha. Junto a este registro a sessão é gravada em áudio ou vídeo. Posteriormente é realizada uma discussão sobre a qualidade da relação terapêutica, as habilidades do terapeuta e sobre as análises funcionais. O supervisor e outros alunos devem fornecer feedback sobre os comportamentos observados. Também é solicitado que o aluno transcreva quinze minutos da sessão e elabore um relatório e uma auto-avaliação do terapeuta e co-terapeuta.

Para aprimorar a supervisão clínica, alguns recursos podem ser utilizados pelo supervisor e supervisionando. Entre eles, o ponto de escuta eletrônico, a observação direta a partir de salas com espelho unidirecional, as gravações de áudio e vídeo e o checklist (Beckert, 2002; Gongora, 1999; Guilhardi, 1988; Mayer et al, 2014; Meyer et al, 2015; Rangé, Guilhardi, Kerbauy, Falcone, \& Ingberman, 1998; Silvares, 1997; Wielenska, 2000). Na utilização destes recursos devem ser considerados aspectos financeiros da instituição, disponibilidade de recursos humanos e espaço apropriado para atividades a serem desenvolvidas. Um ponto a ser destacado é a disponibilidade de verba que a instituição tem para investir com a aquisição e manutenção deste tipo de equipamento (Silvares \& Gongora, 2006).

Independentemente do recurso utilizado, é importante que o aluno passe por um período de treinamento e adaptação para conseguir concentrar-se tanto no relato do cliente quanto nas instruções do supervisor. Tal treinamento poderia ser feito com o auxílio de outros alunos que encenariam o papel de um cliente e somente após a adaptação a tal tecnologia o aluno seria liberado para a atuação direta com o cliente. Estes dois recursos permitem um feedback imediato à atuação do supervisionando, além de permitir uma análise mais acurada do que ocorre na sessão, diferentemente do que ocorre quando o feedback é fornecido a partir da descrição do supervisionando sobre o que ocorreu na sessão (Silvares \& Gongora, 2006).

A sala com espelho unidirecional pode servir para diversos fins. Permite que o supervisor assista à sessão em tempo real, possibilita que outros alunos observem a atuação do colega e aprendam a partir de um modelo. Assim, seria altamente desejável que alunos em treinamento observem pessoas mais experientes atuando na clínica. Além disso, este recurso possibilita o feedback imediato do supervisor aos comportamentos do aluno, além de permitir que este profissional oriente sobre aspectos da sessão mais importantes para serem relatados em supervisão. Tal condição pode aumentar a chance de tatos mais fidedignos quando a observação na sala de espelho por parte do supervisor não for possível. A impossibilidade desta observação constante por parte do supervisor é esperada devido às demandas frequentes das instituições de ensino e à baixa disponibilidade de horários por parte de profissionais que ali atuam. Outra forma de garantir o tato fidedigno na supervisão seria o revezamento de outros alunos na sala de espelho que poderiam auxiliar no momento do relato (Silvares, 1997).

As gravações de áudio e vídeo são alternativas quando a instituição não possui o recurso da sala espelho. Com as gravações o supervisor pode ter contato com o que ocorre em sessão, pode selecionar momentos específicos para analisar e o mais importante, o próprio aluno pode ter acesso ao seu comportamento em sessão e analisar quais comportamentos devem manter-se e quais precisam ser modificados (Meyer et al, 2015).

Alguns alunos podem demonstrar resistência às observações das sessões por parte do supervisor e de seus colegas. Para solucionar tal problema, Silvares (1997) recomenda que sejam feitas algumas atividades envolvendo encenações de atendimentos em que o aluno possa fazer o papel de terapeuta e cliente. Rangé et al (1998) também defendem o uso de vídeos como recurso didático em aulas expositivas para demonstrar exemplos de comportamentos terapêuticos apropriados, argumentando que em condições semelhantes seria mais fácil o aprendiz emitir os mesmos comportamentos. 
A qualidade da supervisão clínica depende da relação entre supervisor e supervisionando (Beckert, 2002; Chippari, Samelo, \& Capelari, 2011; Guilhardi, 1988; Rangé et al, 1998; Vandenberghe, 1997; Vandenberghe, 2014). Considerando tal aspecto, Beckert (2002) aponta que fatores da relação entre supervisor e supervisionando podem ser facilitadores ou obstáculos à formação do aluno. Por isso o autor sugere o enfoque da Psicoterapia Analítico-Funcional (FAP) no contexto de supervisão, salientando a importância de o supervisor observar os comportamentos do supervisionando que são característicos de um terapeuta comportamental, reforçando diferencialmente tais comportamentos e fornecendo modelo de atuação.

A qualidade da relação entre o supervisor e supervisionando também é importante para que este possa fornecer tatos mais acurados em supervisão sobre o que ocorre em sessão (Beckert, 2002; Guilhardi, 1988). Se o aluno entende que pode ser punido pelo professor é provável que edite o relato das sessões, omitindo informações importantes referentes a dúvidas e dificuldades e que passe a relatar somente os conteúdos passíveis de valorização. Por este motivo, é necessário que o supervisor atue como uma audiência não punitiva enquanto o supervisionando relata a sessão. Ademais, a relação aqui enfatizada pode servir como modelo para o aluno de como criar uma relação com seu cliente de modo que beneficie a condução do caso clinico.

Contudo, ao analisar as contingências que vigoram em uma supervisão clínica, Vandenberghe (1997) defende a ideia de que a experiência se aproxima mais de um contexto terapêutico do que educacional. $\mathrm{O}$ autor aponta três contextos distintos que envolvem supervisão; no primeiro, o supervisor estaria preocupado em ser "terapeuta" do cliente atendido por seu supervisionando. Neste caso, daria instruções ao aluno sobre o que fazer para que o cliente tenha um bom prognóstico. Agindo desta forma o supervisor não estaria ensinando o aluno a ser terapeuta ou agir de modo independente, pois seu foco seria a melhora do cliente. No segundo contexto, o foco estaria em ser o "terapeuta" do aluno, modelando comportamentos importantes para a atuação clínica. Por fim, no terceiro contexto o supervisor se preocuparia com a relação terapêu- tica entre aluno e cliente. O foco, neste caso, seria analisar e deixar claro para o aluno como variáveis da relação afetam tanto o cliente como o terapeuta e o que é possível mudar para que a relação melhore, aprimorando assim a condução do caso.

Sobre este aspecto ressalta-se o papel do supervisor na condução dos casos atendidos pelos alunos. A compreensão que o aprendiz desenvolve sobre sua relação com o paciente pode transcorrer também da interação verbal entre ele e seu supervisor, isto é, o supervisor apreende o relato verbal do supervisionando para assessora-lo na compreensão de elementos do processo terapêutico de seus pacientes (Wielenska, 2000). Por esta razão, o comportamento do aluno de analisar deve receber destaque também durante as supervisões.

Meyer et al (2015) apresentaram a condução de um grupo de supervisão, em que o foco principal foi desenvolver o comportamento de analisar. Os autores também dão importância aos sentimentos do terapeuta em sessão e na supervisão. AbreuMotta, et al. (2010) também defendem a análise dos encobertos e apontam que evocar os sentimentos e pensamentos em supervisão é imprescindível para o sucesso da terapia. Assim, ao investigar sobre os encobertos do terapeuta/supervisionando seria possível alcançar variáveis importantes para a condução do caso clinico.

Tanto para a formação do aluno, quanto para a condução de casos clínicos, ressalta-se a importância da literatura sobre a tomada de decisão frente às demandas analisadas (Meyer, et al., 2015). Portanto, é crucial que os terapeutas estejam familiarizados com atividades de busca em fontes de informação sobre aspectos dos casos que atendem. Tal habilidade apresentada pode ser desenvolvida em diversas disciplinas, inclusive nas disciplinas iniciais da graduação, uma vez que fazer busca em base de dados científicas é uma habilidade necessária para a atuação dos profissionais que querem estar atualizados sobre os progressos na área de psicoterapia baseada em evidências.

O aluno deve ser preparado para uma atuação em que nem sempre poderá ter o feedback de terceiros sobre seu desempenho. Por isso é fundamental o desenvolvimento de autonomia. Para isto, as supervisões devem ser menos diretivas para que o próprio aluno possa fazer suas análises 
e tomar decisões quanto à condução do caso que atende (Silvares, 1997).

Geralmente o atendimento clínico supervisionado é feito a partir do serviço oferecido em clínica-escola. Contudo, alerta-se para o fato de que a população que procura este serviço nem sempre é a mesma que o aluno terá contato após sua formação. Neste sentido, Guilhardi (1998) faz uma reflexão quanto ao repertório desenvolvido na graduação e o repertório exigido no contexto da clínica particular. Tais diferenças podem acarretar problemas quanto à generalização das habilidades desenvolvidas durante a formação e as habilidades exigidas após a mesma. Na graduação o aluno está sob controle de carga horária a ser cumprida, tem o respaldo de um supervisor e da instituição onde está matriculado. Em condições reais, após a conclusão da graduação, outra variável a ser incluída é o valor das sessões e todo o enquadre característico de atendimento em clínica privada, como o procedimento em caso de faltas, férias, frequência e a duração das sessões. Essas variáveis, embora tratadas de modo teórico, em aulas expositivas, na graduação, não fazem parte da vivência do supervisionando no atendimento em clínica-escola.

Algumas clínicas-escola de universidades particulares têm cobrado dos clientes um valor simbólico de pagamento das sessões, muito abaixo do valor de mercado. Esse critério possibilita que o aluno exercite o comportamento de informar e receber o dinheiro a ser pago pelo cliente, comportamento este que será frequente nas atividades futuras deste aluno. No entanto, o pagamento de valor simbólico não pode ser considerado um equivalente funcional ao honorário do profissional uma vez que as consequências do não pagamento de tais valores são diferentes, pois os serviços da clínica-escola nas instituições públicas são financiados pelo Sistema Único de Saúde o que torna a contribuição facultativa.

\section{Considerações finais}

No presente artigo apresentou-se aspectos importantes do desenvolvimento da Terapia AnalíticoComportamental no Brasil, articulando esse processo aos acontecimentos sócio-históricos no país. Percebe-se ainda a necessidade de estudos que in- vestiguem o papel de diversas variáveis na formação de terapeutas, demonstrando os recursos mais eficientes, disciplinas que devem fazer parte das grades curriculares e fatores necessários para melhorar a formação de terapeutas comportamentais.

Espera-se que o artigo seja útil para iniciantes na clínica analítico-comportamental e sirva também como recurso didático para professores de disciplinas de Análise do Comportamento na graduação de universidades brasileiras. Recomendam-se novos estudos para comparar a eficácia dos métodos de supervisão, produzindo dados baseados em evidências que permitam investimentos produtivos no treinamento do analista do comportamento.

\section{Referências}

Abreu-Motta, H. O., De-Farias, A. K. C. R., \& Coelho, C. (2010). Habilidades terapêuticas: é possível treiná-las. Em: A.K.C.R. De-Farias (Org). Análise Comportamental Clínica: aspectos teóricos e estudos de caso, (pp.49-65). Porto Alegre: Artmed.

Barcellos, A. B. \& Haydu, V.B. (1998). História da Psicoterapia Comportamental. Em B. Rangé (Org.) Psicoterapia Comportamental e Cognitiva: Pesquisa, Prática, Aplicações e Problemas (pp. 43-53). Campinas, SP: Editorial Psy Ltda.

Beckert, M. (2002). Relação supervisor-supervisionando e a formação do terapeuta: contribuições da psicoterapia analítico-funcional (FAP). Em: H. J. Guilhardi, M. B. B. P. Madi, P. P. \& Queiroz. M. C. Scoz (Orgs.). Sobre Comportamento e Cognição: contribuições para a construção da teoria do comportamento, (pp.245-256). Santo André: ESETec.

Botomé, S. P. (2006). Contribuições, Participação, Organização e Representação da Análise Experimental do Comportamento nos Eventos e na Organização da Psicologia no Brasil: A ABPMC como e ponto de partida. Revista Brasileira de Terapia Comportamental e Cognitiva, 6(2), 217-231.

Callaghan, G. M., Duenas, J. A., Nadeau, S. E., Darrow, S. M., Merwe, J. V., \& Misko, J. (2012). An Empirical Model of Body Image Disturbance Using Behavioral Principles 
found in Functional Analytic Psychotherapy, Acceptance and Commitment Therapy. International Journal of Behavioral Consultation Therapy, 7(2), 16-24.

Carrara, K. (2005). Behaviorismo radical: crítica e metacrítica. São Paulo: Ed. UNESP.

Cattivelli, R., Tirelli, V., Berardo, F., \& Perin, S. (2012). Promoting Appropriate Behavior in Daily Life Contexts Using Functional Analytic Psychotherapy in Early-Adolescent Children. International Journal of Behavioral Consultation Therapy, 7(2), 25-32.

Cesar, G. (2002). Análise do Comportamento no Brasil: Uma revisão histórica de 1961 a 2001, a partir de publicações. Dissertação de Mestrado, Programa de Pós-Graduação em Psicologia Experimental: Análise do Comportamento, Pontifícia Universidade Católica de São Paulo.

Chippari, M., Samelo, M. J., \& Capelari, A. (2011). Ensino da Análise do Comportamento e Desdobramentos: A relação Professor-Aluno e Variáveis que Influenciam a Aprendizagem. Em C.V.B.B. Pêssoa, C.E. Costa \& M.F. Benvenuti. (Orgs). Comportamento em Foco (Volume 1), (pp. 125-130). São Paulo, SP: Associação Brasileira de Psicologia e Medicina Comportamental - ABPMC.

Conte, F. C. D. S. (2010). Reflexões sobre o sofrimento humano e a Análise Clínica Comportamental. Temas em Psicologia, 18(2), 385-398.

D’Zurilla, T. J. \& Goldfried, M.R. (1971). Problem Solving and Behavior Modification. Journal of Abnormal Psychology, 78(1), 107-126.

Falcone, E. (1999). A avaliação de um Programa de Treinamento da Empatia com Universitários. Revista Brasileira de Terapia Comportamental e Cognitiva, 1(1), 23-32.

Follette, W. C., Naugle, A. E., \& Callaghan, G. M. (1996). A Radical Behavioral Understanding of the Therapeutic Relationship in Effecting Change. Behavior Therapy, 27(4), 623-641.

Gongora, M. A. (1999). Aprendendo Entrevista Clínica Inicial: Contribuições para a Formação do Terapeuta. Em: R.A. Banaco (Org.), Sobre Comportamento e Cognição: aspectos teóricos, metodológicos e de formação em análise do comportamento e terapia cognitivista, (pp.516-524).
Santo André-SP: ARBytes.

Guilhardi, H. J. (1988). A Formação do Terapeuta Comportamental. Que Formação? Em: H. W. Lettner \& B. P. Rangé (Orgs.), Manual de Psicoterapia Comportamental (pp.313-320). São Paulo: Manole.

Guilhardi, H. J. (2001). Com que contingências o terapeuta trabalha em sua atuação clínica. Em: R. A. Banaco (Org), Sobre Comportamento e Cognição: aspectos teóricos, metodológicos e de formação em Análise do Comportamento, (pp. 316-331). Santo André: ESETec.

Gorayeb, R. (1990). Conheça o Histórico da SBP. http://novo.sbponline.org.br. Acedido dezembro 28, 2015. http://novo.sbponline.org.br/ conheca-o-historico-da-sbp-relatado-por-ricardo-gorayeb-e-reinier-rozestraten

Hayes, S. C. (1987). A contextual approach to therapeutic change. In N. Jacobson (Ed.), Psychotherapists in clinical practice: Cognitive and behavioral perspectives (pp. 327-387). New York: Guilford Press.

Hayes, S. C., Strosahl, K. D., \& Wilson, K. G. (1999). Acceptance and commitment therapy: An experiential approach to behavior change. Nova York: Guilford Jacobson, N. S. (1992). Behavioral couple therapy: A new beginning. Behavior Therapy, 23, 493-506.

Kazdin, A. E. (1985). History of Behavior Modification. Em: A. S. Bellack, M. Hersen, \& A.E. Kazdin (Orgs.) International Handbook of Behavior Modification and Therapy. New York: Plenum.

Kohlenberg, R. J., \& Tsai, M. (2001). Psicoterapia Analítica Funcional (F. Conte, M. Delliti, M. Z. Brandão, P. R. Derdyk, R. R. Kerbauy, R. C. Wielenska, R. A. Banaco, R. Starling, trads.). Santo André, SP: ESETEc (Obra publicada originalmente em 1991).

Lindsley, O., Skinner, B. F., Solomon, H. C. (1953). "Studies in Behavior Therapy (Status Report I)". Walthama, MA.: Metropolitan State Hospital.

Linehan, M. M. (1993). Cognitive-behavioral treatment of borderline personality disorder. New York: Guilford.

Mattana, P. E. (2004). Comportamentos Profissionais do Terapeuta Comportamental como Objetivos para sua Formação. Dissertação de Mestrado, 
Programa de Pós-Graduação em Psicologia, Universidade Federal de Santa Catarina, Florianópolis,SC.

Mayer, A. P. F, Nascimento, M. R. D., Sartor, M. S., Sabbag, G. M., Barbosa, R. M. M., Brandenburg, O. J., Yingberman, Y. K. \& Maly Delitti, M. (2014). Uma Proposta de Treinamento de Habilidades Terapêuticas na Formação de Terapeutas Analítico-Comportamentais. Em: C. Vichi, E.Huziwara, H. Sadi \& L. Postalli. (Orgs.). Comportamento em Foco (Volume 3) (pp. 223-236). São Paulo, SP: Associação Brasileira de Psicologia e Medicina Comportamental ABPMC.

Meyer, S. B. \& Vermes, J S (2001). Relação Terapêutica Em: B. Rangé (Org). Psicoterapias Cognitivo-Comportamontais: um diálogo com a psiquiatria (pp 101-110). Porto Alegre, RS: Artmed.

Meyer, S. B., Villas-Bôas, A. A.., Franceschini, A. C. T., Oshiro, C. K. B., Kameyama, M., Rossi, P. R., Mangabeira, V. (2015). Terapia AnalíticoComportamental: Relato de Casos e de Análises (Volume 1). São Paulo: Núcleo Paradigma.

Millenson, J. R. (1975). Princípios de Análise do Comportamento. Brasília: Coordenada.

Moreira, S. B. S. (2003). Descrição de Algumas Variáveis em um Procedimento de Supervisão de Terapia Analítica do Comportamento. Psicologia: Reflexão e Crítica, 16(1), 157-170.

Neno, S. (2003). Análise funcional: Definição e aplicação na terapia analítico-comportamental. Revista brasileira de terapia comportamental e cognitiva, 5(2), 151-165.

Oshiro, C. K. B., Kanter, J., \& Meyer, S. B. (2012). A Single-Case Experimental Demonstration of Functional Analytic Psychotherapy with Two Clients with Severe Interpersonal Problems. International Journal of Behavioral Consultation Therapy, 7(2), 111-116.

Palhoco, A. R. D. M. S. (2011). Estudo da Empatia e da Percepção de Emoções em Psicoterapeutas e Estudantes de Psicologia. Dissertação de Mestrado, Programa de Pós-Graduação em Psicologia, Universidade de Lisboa, Portugal.

Pavlov, I. P. (1928). Lectures on conditioned reflexes. New York: International Publishers.

Pezzato, F. A., Brandão, A. S., \& Oshiro, C. K. B.
(2012). Intervenção Baseada na Psicoterapia Analítica Funcional em um Caso de Transtorno de Pânico com Agorafobia. Revista Brasileira de Terapia Comportamental e Cognitiva, 15 (1), 74-84

Prado, O. Z., \& Meyer, S. B. (2004). Relação Terapêutica: A Perspectiva Comportamental, Evidências e o Inventário de Aliança de Trabalho (WAI). Revista Brasileira de Terapia Comportamental e Cognitiva, 6(2), 201-209.

Rangé, B., Guilhardi, H. J., Kerbauy, R.R., Falcone, E. M. O., \& Ingberman, Y.K. (1998) Ensino, Treinamento e Formação em Psicoterapia Comportamental e Cognitiva. Em: B. Rangé (Org.) Psicoterapia Comportamental e Cognitiva: Pesquisa, Prática, Aplicações e Problemas (pp. 331-351). Campinas, SP: Psy.

Richelle, M. N. (1995). BF Skinner: a reappraisal. Bélgica: Psychology Press.

Rozestraten, R. (1988). "Os Primórdios da SBP" (mais sobre a história). http://novo.sbponline.org.br. Acedido dezembro 28, 2015. http:// novo.sbponline.org.br/conheca-o-historico-da-sbp-relatado-por-ricardo-gorayeb-e-reinier-rozestraten

Rubiano, M. R. B. (n.d.). Apresentando a Sociedade Brasileira de Psicologia. http://novo.sbponline. org.br. Acedido dezembro 28, 2015. http://novo. sbponline.org.br/marcia-regina-bonagamba-rubiano-apresenta-a-sbp

Sant'Anna, R. \& Gongora, M. (1987). Por uma Postura Behaviorista no Contexto Clínico. In: III Encontro de Terapeutas Comportamentais. Brasília, inédito.

Silvares, E. F. M. (1991). A Evolução do Diagnóstico Comportamental. Psicologia: Teoria e Pesquisa, 7(2), 179-187.

Silvares, E. F. M. (1997). Dificuldades na Graduação e Pós-Graduação, com a Prática Clínica Comportamental. Em: R. A. Banaco (Org.), Sobre comportamento e Cognição: aspectos teóricos, metodológicos e de formação em análise do comportamento e terapia cognitivista (pp 503510). Santo André: ARBytes.

Silvares, E.F.M. \& Range, B. (2001). Avaliação e Formulação de Casos Clínicos Adultos e Infantis. Em: B. Range (Org.) Psicoterapias Cognitivo Comportamentais: Um Diálogo com 
a Psiquiatria (pp.79-100). Porto Alegre, RS: Artmed.

Silvares, E. F. M, \& Gongora, M. A. N. (2006). Psicologia Clínica Comportamental: A Inserção da Entrevista com Adultos e Crianças. São Paulo, SP: Edicon.

Skinner, B. F. (1935). Two types of conditioned reflex and a pseudo type. The Journal of General Psychology, 12(1), 66-77.

Skinner, B. F. (1937). Two types of conditioned reflex: A reply to Konorski and Miller. The Journal of General Psychology, 16(1), 272-279.

Souza, D. M., \& Alves, Z. M. M. B. (1988). Anais da XVIII RA, 3-4.

Thorndike, E. L. (1898). Animal intelligence: an experimental study of the associative processes in animals. The Psychological Review: Monograph Supplements, 2(4), 1.

Todorov, J. C. \& Hanna, E. S. (2010). Análise do Comportamento no Brasil. Psicologia: Teoria e Pesquisa, 26 (Número Especial), 143-153.

Tourinho, E. Z. (1999). Estudos Conceituais na Análise do Comportamento. Temas em Psicologia, 7(3), 213-222.

Ulian, A. L. A. D. O. (2007). Uma sistematização da prática do terapeuta analítico-comportamental: subsídios para a formação. Tese de Doutorado, Instituto de Psicologia, Universidade de São Paulo, São Paulo.

Vandenberghe, L. (1997). Uma abordagem contextual da supervisão clínica. Sobre Comportamento e Cognição, 1 (1), 332-340.

Vandenberghe, L. (2002). A prática e as implicações da análise funcional. Revista Brasileira de Terapia Comportamental e Cognitiva, 4(1), 3545.

Vandenberghe, L. (2007). Terapia comportamental construtiva: uma outra face da clínica comportamental. Psicologia USP, 18(4), 89-102.

Vandenberghe, L. (2014). Supervisão para terapia comportamental. Em: Haydu, V. B.; Fornazari, S. A. \& Estanislau, C. R. (Orgs.) Psicologia e Análise do Comportamento: Conceituações e Aplicações à Educação, Organizações, Saúde e Clínica (pp 477-495). Londrina: Universidade Estadual de Londrina.

Watson, J. B. (1913). Psychology as the behaviorist views it. Psychological review, 20(2), 158.
Wielenska, R. C. (2000). A investigação de alguns aspectos da relação terapeuta-cliente em sessões de supervisão. Revista Brasileira de Terapia Comportamental e Cognitiva, 2(1), 09-19.

Witter, G P. (2006). Supervisor-Estagiário-Cliente: Destinatários de Nossas Intervenções. Em: C. Ramos, G. G. Silva, \& S. Souza (Orgs.) Práticas Psicológicas em Instituições: Uma Reflexão sobre os Serviços-Escola (pp. 200-206). São Paulo: Vetor.

Kazdin, A. E. (1985). History of Behavior Modification. Em: A. S. Bellack, M. Hersen, \& A.E. Kazdin (Orgs.) International Handbook of Behavior Modification and Therapy. New York: Plenum.

\section{Informações do Artigo}

Histórico do artigo:

Submetido em: 05/01/2016

Primeira decisão editorial: 25/03/2016

Segunda decisão editorial: 03/06/2016

Aceito em 01/07/2016

Editor associado: Nicodemos Batista Borges 